\title{
Crack shielding in non-planar and frictional discontinuities under mixed-mode loading
}

\author{
Andrea Spagnoli ${ }^{1}$, Andrea Carpinteri, and Michele Terzano \\ Department of Engineering and Architecture, Università di Parma, Parco Area delle Scienze 181/A, \\ 43124 Parma, Italy
}

\begin{abstract}
In this paper, a two-dimensional model to describe the nonplanar features of crack morphologies is presented. The model accounts for frictional tractions along the crack surfaces by considering an elasticplastic-like constitutive interface law. Dilatancy effect due to crack roughness is described by the model, leading to a Mode I/II coupling between displacements and tractions along the crack surfaces. The nonlinear solution of the rough and frictional crack under general remote scenarios is obtained using the Distributed Dislocation Technique (DDT). By considering a linear piecewise periodic profile of the interface crack, the influence of roughness and friction of interface cracks is examined in relation to both the resulting near-tip stress field and the fracture resistance under monotonic mixed-mode loading. The present model is able to quantify the increase of the fracture resistance due to roughness- and friction-induced crack tip shielding and to correlate it with a dimensionless crack size parameter.
\end{abstract}

\section{Introduction}

Traction-free and planar cracks represent a rather idealized picture of the physical reality, commonly used in fracture mechanics problems. As a matter of fact, experimental observations of the cracking behaviour of hard brittle materials, such as concrete and rocks, but also bone and glassy polymers, showed that the real picture is much more complicated. For instance, failure of structural components under shear loading may occur at larger critical loads than those predicted by Linear Elastic Fracture Mechanics (LEFM); furthermore, the crack path kinks with respect to the direction predicted and its interfaces appear rough and irregular. Crack kinking is a result of dilatancy, a phenomenon caused by the interaction between tangential and normal displacement components along rough surfaces. In order to account for the actual features of crack surfaces, the first two authors have recently explored the influence of crack path meandering on fatigue propagation, by modelling the crack profile as a piecewise linear curve in two dimensions $[1,2]$ (the same type of model was initially conceived in the realm of fractal geometry [3]).

In the present paper, the influence of roughness and friction of two-dimensional cracks is examined in relation to both the resulting near-tip stress field and the fracture resistance 
under monotonic mixed-mode loading, analysing different combinations of the Mode I and Mode II loadings. A non-linear model is presented where an elastic-plastic-like constitutive interface law [4] is adopted to describe the Mode I/II coupling between displacements and tractions along the crack surfaces. Positive values of Mode I relative displacement, corresponding to a separation condition, are locally permitted along the crack profile by such a model. The solution of the crack problem is obtained using the Distributed Dislocation Technique (DDT) [5]. By considering a linear piecewise periodic profile of the interface crack, the present model is employed to quantify the increase of the fracture resistance due to crack tip shielding (e.g. see [6]) for given values of the relative crack size, measured as the ratio between the crack length and the periodic length of the crack roughness, and various loading scenarios.

\section{The model of a non-planar and frictional crack}

Crack surfaces interference is a result of several factors, such as the presence of microstructural inhomogeneities, residual stresses, material properties dispersion, multiaxial remote stresses. A simple interface two-dimensional model, considering the effects of friction and roughness along the crack, has recently been presented by the authors $[5,7,8]$. Such a model is formulated as a constitutive relationship written in the form of a classic elastic-plastic law with non-associative flow rule. The crack is assumed as globally smooth, with the Coulomb's friction and the surface roughness embedded in the constitutive formulation. A periodic zig-zag pattern of the crack profile is adopted, characterized by a constant kink angle $\alpha$, mean length of the asperities equal to $d$ and height $h$, see Fig. 1a.

The elastic-plastic relationship is conveniently written in terms of relative displacement increments and bridging stresses between two opposing points of the crack. The increment of relative displacement is composed of a recoverable elastic part $d w_{i}^{e}$, related to the remote stress field, and a non-recoverable plastic part $d w_{i}^{p}$, which accounts for frictional sliding and dilatancy. The stresses on the crack interface are related to the displacement increments by means of interface stiffness $E_{i j}^{E P}$ (summation convention is applied to repeated indexes):

$$
d \sigma_{i}^{(b)}=E_{i j}^{E P} d w_{j} \quad i, j=t, n
$$

where $t, n$ denote, respectively, the tangential and normal directions related to the nominally flat crack surface (Fig. 1a). The non-associative flow rule is described by a slip function $F$ and a slip potential $G$ :

$$
\begin{aligned}
& F=\left|\sigma_{1}^{(b)}\right|+m \sigma_{2}^{(b)}=\left|\sigma_{n}^{(b)} \sin \alpha+\sigma_{t}^{(b)} \cos \alpha\right|+m\left(\sigma_{n}^{(b)} \cos \alpha-\sigma_{t}^{(b)} \sin \alpha\right) \\
& G=\left|\sigma_{1}^{(b)}\right|=\left|\sigma_{n}^{(b)} \sin \alpha+\sigma_{t}^{(b)} \cos \alpha\right|
\end{aligned}
$$

where $m$ is the coefficient of the Coulomb's friction. The non-recoverable displacement increment is written as

$$
d w_{i}^{p}= \begin{cases}0 & \text { if } F<0 \text { or } d F=0 \\ \lambda \frac{\partial G}{\partial \sigma_{i}^{(b)}} & \text { if } F=d F=0\end{cases}
$$


while the interface stiffness $E_{i j}^{E P}$ is computed as follows:

$$
E_{i j}^{E P}=\left\{\begin{array}{c}
E_{i j} \quad \text { if } F<0 \text { or } d F=0 \\
E_{i j}-\frac{\frac{\partial F}{\partial \sigma_{p}^{(b)}} E_{i q} E_{p j} \frac{\partial G}{\partial \sigma_{q}^{(b)}}}{\frac{\partial F}{\partial \sigma_{p}^{(b)}} E_{p q} \frac{\partial G}{\partial \sigma_{q}^{(b)}}} \text { if } F=d F=0
\end{array}\right.
$$

where $E_{i j}$ is the elastic interface stiffness. If the normal relative displacement is positive, i.e. corresponding to a separation condition of the crack surfaces, the interface stiffness is assumed to be null.

This formulation of the interface model is particularly amenable for a direct implementation in numerical methods, such as the DDT, which is one of the so-called displacement discontinuity methods, commonly employed in the solution of the integral equations with singular terms, arising in several crack problems. We introduce a suitable distribution of climb and glide dislocations along the crack surface, representing opening and sliding displacement discontinuities between the crack surfaces, respectively. The stresses, resulting from such discontinues in a continuous body, are known as corrective stresses and present the following form (see Fig. $1 \mathrm{~b}$ for an explanation of the variables):

$$
\bar{\sigma}_{i}(x)=\frac{2 \mu}{\pi(\kappa+1)} \int_{0}^{a} \frac{B_{i}(\xi)}{x-\xi}+B_{j}(\xi) F_{i j}(x, \xi) d \xi \quad i, j=t, n
$$

$\mu$ is the elastic shear modulus, $\kappa$ is the material Kolosov constant, whereas $F_{i j}$ are influence functions which account for the specific geometry of the problem, whose expression for the case considered here can be found in the literature [9]. $B_{i}(\xi)$ is the dislocation density $\left(B_{i}(\xi)=d b_{i}(\xi) / d \xi\right.$, with $b_{i}=$ Burger vector), which yields the relative displacement between the crack surfaces by integration.

The corrective stresses are superposed on the stresses generated by the remote loads to obtain the overall stress state along the crack. Notice that if the applied loads are expressed in terms of nominal SIFs $K_{I}$ and $K_{I I}$ (Fig. 1b), we have $\sigma_{n}^{(\infty)}=K_{I} /\left(Y_{I} \sqrt{\pi a}\right)$ and $\sigma_{t}^{(\infty)}=K_{I I} /\left(Y_{I I} \sqrt{\pi a}\right)$, where $Y_{I}, Y_{I I}$ are geometric factors. In the realm of LEFM, the overall stress state on the crack surfaces would be null; here, on the contrary, we introduce the bridging stresses resulting from the surface interaction. The integral equation resulting from equilibrium takes the following form:

$$
\sigma_{i}^{(\infty)}(x)+\frac{2 \mu}{\pi(\kappa+1)} \int_{0}^{a} \frac{B_{i}(\xi)}{x-\xi}+B_{j}(\xi) F_{i j}(x, \xi) d \xi=\sigma_{i}^{(b)}(x) \quad i, j=t, n
$$

Following the standard method of solution for integral equations, we normalise the interval between $[-1,1]$ and replace the variables $x, \xi$ with $u, v$, respectively. Then, we express the unknown dislocation densities as follows:

$$
B_{i}(u)=\phi_{i}(u) \omega(u)=\phi_{i}(u) \sqrt{\frac{1+u}{1-u}} \quad i=t, n
$$


where $\omega(u)$ is the fundamental singular function, suitable for an edge crack, whilst $\phi_{i}(u)$ are the unknowns. Using the Gauss-Chebyshev numerical quadrature, the integral of Eq. (6) is converted in a set of non-linear algebraic equations. An efficient incremental solution of the non-linear problem is achieved by using the Newton-Raphson technique with an iteration control based on displacement increments and residuals.

At each load increment, the effective Mode I and Mode II stress intensity factors at the crack tip are computed from the unknown functions $\phi_{i}$, through an extrapolation to the singular point $u=+1$ :

$$
k_{I}=\frac{2 \mu}{\pi(\kappa+1)} \sqrt{2 \pi a} \phi_{n}(1), \quad k_{I I}=\frac{2 \mu}{\pi(\kappa+1)} \sqrt{2 \pi a} \phi_{t}(1)
$$

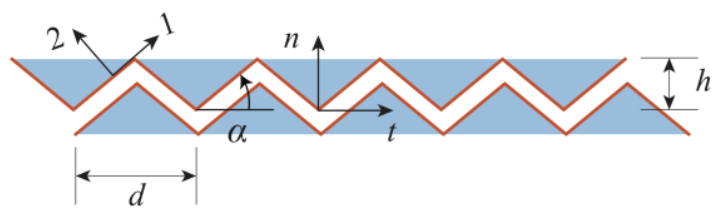

(a)

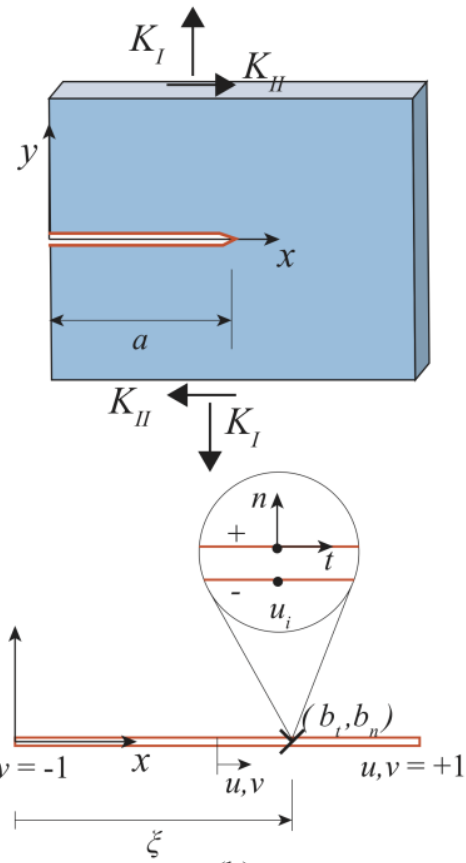

(b)

Fig. 1. (a) The saw-tooth asperity model, showing the local and global reference systems adopted in the formulation, Eqs (1)-(4); (b) Schematic model of the geometry with an edge crack of length $a$.

\section{Crack shielding under mixed mode loading}

The specific case of an infinite plate containing an edge crack of length $a$, exposed to remote compressive load and shear load respectively, is considered. The tractions are assumed to increase proportionally up to the maximum values $\sigma_{\max }$ and $\tau_{\max }$, so that the corresponding nominal SIFs (Fig. 1b) are expressed as follows:

$$
K_{I}=-1.12 \sigma_{\max } f(t) \sqrt{\pi a}, K_{I I}=1.12 \tau_{\max } f(t) \sqrt{\pi a}
$$


where $f(t)$ is a time function, with $0 \leq f(t) \leq 1$. Note that the Mode I SIF $K_{I}$ is negative due to the compressive load applied. The load angle is expressed by $\beta=\arctan \left(-K_{I I} / K_{I}\right)=\arctan \left(-\tau_{\max } / \sigma_{\max }\right)$.

At each load increment, the effective Mode I and Mode II SIFs $k_{I}$ and $k_{I I}$ (see Eq. 8) are calculated according to the model described in the previous section. Then, the critical condition of fracture is obtained by means of the classical criterion of the Maximum Tangential Stress (MTS) [10] (some results and discussions on mixed-mode crack growth under fatigue loading can be found for instance in [11-13]). Accordingly, the propagation angle of the crack is given by

$$
\theta_{c}=2 \arctan \left[\frac{1}{4} \frac{k_{I}}{k_{I I}} \pm \frac{1}{4} \sqrt{\left(\frac{k_{I}}{k_{I I}}\right)^{2}+8}\right]
$$

and the critical condition of fracture by

$$
K_{I c}=k_{I} \cos ^{3}\left(\frac{\theta_{c}}{2}\right)-3 k_{I I} \cos ^{2}\left(\frac{\theta_{c}}{2}\right) \sin \left(\frac{\theta_{c}}{2}\right)
$$

where $K_{I c}$ is the Mode I fracture toughness of the material. Note that the MTS criterion has been conceived for positive values of Mode I SIF, so that $k_{I}$ is taken to be null in Eqs. (10) $-(11)$ if $k_{I}<0$.

Let us discuss the effect of crack shielding due to friction and roughness of the crack by applying the present model. The load angle $\beta$ is varied between $0^{\circ}$ to $90^{\circ}$ (corresponding to pure Mode II loading). The characteristic length of the crack roughness is taken equal to $d=10^{-3} a$, the asperity angle $\alpha$ is varied from 0 (smooth crack) to $10^{\circ}$, and the coefficient of Coulomb's friction is $m=0.5$.

In Figure 2, the evolution of the normalized effective Mode II SIF is shown for different values of the load angle $\beta$; a non-linear trend is observed in the case of rough cracks.

By considering the MTS criterion of Eqs. (10)-(11), the critical condition of fracture can be calculated in terms of the nominal Mode II SIF $K_{I I}$. Figures 3 and 4 illustrate the fracture resistance in terms of the normalized SIF $K_{I I} / K_{I c}$ against the load angle $\beta$ under different levels of crack roughness (see angle $\alpha$ ), along with the values of the propagation angle $\theta_{c}$. As a reference, in the charts it is reported the value of the angle $26.6^{\circ}$ equal to $\arctan (m)$, together with the propagation angle $\theta_{c}=70.5^{\circ}$ related to the pure Mode II loading. The non-monotonic trend of results shown in Figs 3 and 4 for rough cracks can be interpreted by considering the competing shielding effects of friction and dilatancy on fracture resistance.

\section{Concluding remarks}

In the present paper, we have illustrated an application of the Distributed Dislocation Technique (DDT) to describe the near-tip stress fields of two-dimensional cracks characterised by non-planar profiles. The model introduced has allowed us to account for the effects of friction and roughness between crack surfaces, by means of a non-associative, elastic-plastic-like interface law. 
We have applied such model to investigate the crack shielding effect, due to roughness and friction, on the fracture resistance under remote mixed-mode loading. Specifically, we have considered an edge-crack exposed to a remote compressive load combined with a proportional shear load. Some simulations have been carried out to illustrate crack shielding in terms of nominal Mode II SIF against the mode-mixity load angle, considering different degrees of roughness (in terms of the asperity angle). In particular, it appears that crack shielding due to frictional effects is enhanced by the phenomenon of dilatancy, which gives rise to a local opening stress intensity factor. This last feature has been put in evidence through the computation of the kinking angle according to a suitable mixed-mode propagation criterion.

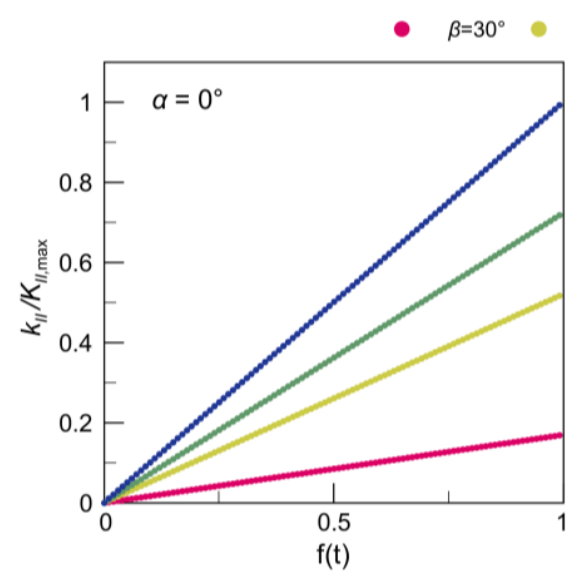

(a)

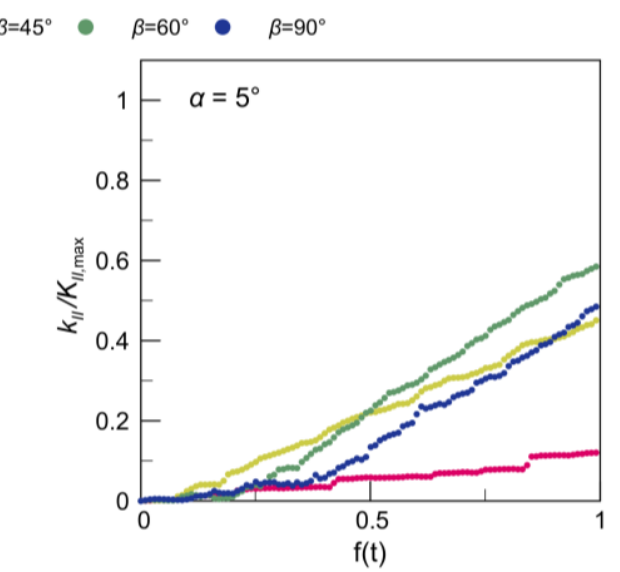

(b)

Fig. 2. Evolution of the effective normalized Mode II SIF for different mixed-mode phase angle $\beta$ : (a) smooth crack $(\alpha=0)$; (b) rough crack with $\alpha=5^{\circ}$.

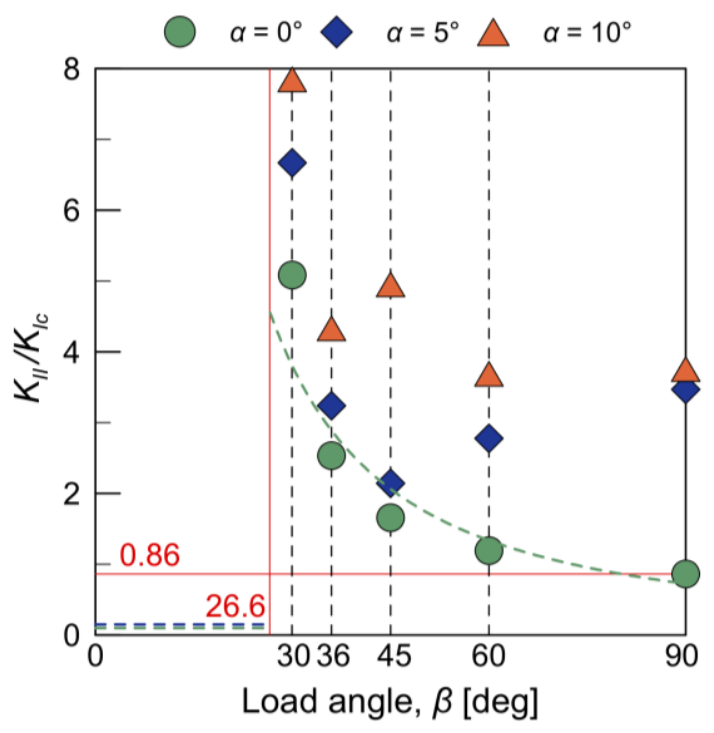

Fig. 3. Effective normalized Mode II SIF at fracture against load angle for different levels of the crack roughness. 


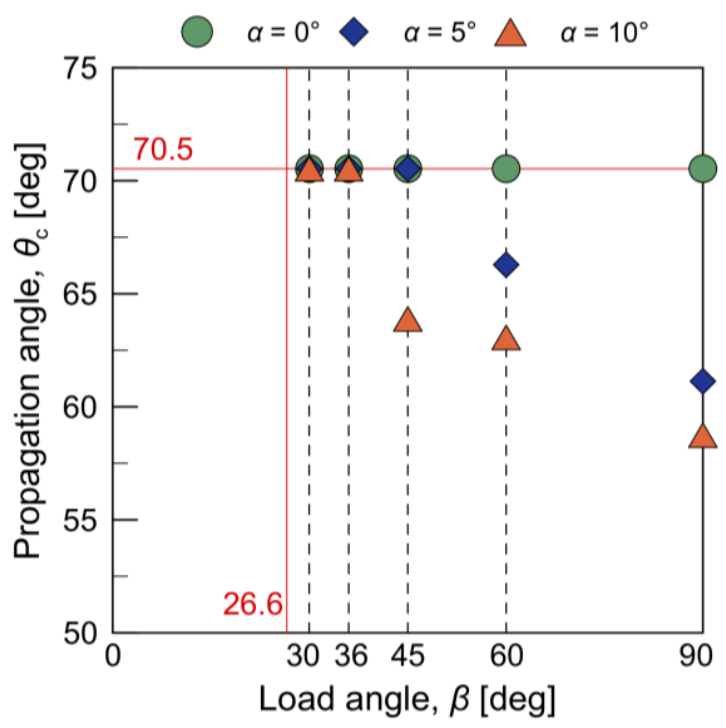

Fig. 4. Propagation angle at fracture against load angle for different levels of the crack roughness.

\section{References}

1. A. Spagnoli, S. Vantadori, A. Carpinteri, Interpreting some experimental evidences of fatigue crack size effects through a kinked crack model. Fatigue and Fracture of Engineering Materials and Structures, 38: 215-222 (2015)

2. R. Brighenti, A. Carpinteri, A. Spagnoli, Influence of material microvoids and heterogeneities on fatigue crack propagation Acta Mechanica, 225: 3123-3135 (2014)

3. A. Carpinteri, A. Spagnoli, S. Vantadori, D. Viappiani, Influence of the crack morphology on the fatigue crack growth rate: A continuously-kinked crack model based on fractals. Engineering Fracture Mechanics, 75: 579-589 (2008)

4. M.E. Plesha, R. Ballarini, The effects of crack surface friction and roughness on crack tip stress fields. Int J Fract, 34: 195-207 (1987).

5. A. Spagnoli, A. Carpinteri, M. Terzano, Near-tip stress fields of rough and frictional cracks under mixed-mode loading. Fatigue \& Fracture of Engineering Materials \& Structures, 41: 2099-2109 (2018)

6. A.G. Evans, J.W. Hutchinson, Effects of non-planarity on the mixed mode fracture resistance of bimaterial interfaces. Acta Metallurgica, 37: 909-916 (1989)

7. A. Carpinteri, A. Spagnoli, M. Terzano, S. Vantadori, Fracture toughness of rough and frictional cracks emanating from a re-entrant corner. Frattura ed Integrità Strutturale, 11: 175-182 (2017)

8. A. Spagnoli, A. Carpinteri, M. Terzano, Size effect on the fracture resistance of rough and frictional cracks. Frattura ed Integrità Strutturale, 13: $401-407$ (2019)

9. D.A. Hills, P.A. Kelly, D.N. Dai, A.M. Korsunsky, Solution of crack problems: the distributed dislocation technique, 44, Springer Science \& Business Media (2013)

10. F. Erdogan, G.C. Sih, On the crack extension in plates under plane loading and transverse shear. Journal of Basic Engineering, 85: 519-525 (1963) 
11. E. Macha, D. Rozumek, Fatigue crack growth in $18 \mathrm{G} 2 \mathrm{~A}$ steel under mixed mode I+ III loading. In ECF15 Proceedings, Stockolm (2004)

12. D. Rozumek, E. Macha, A survey of failure criteria and parameters in mixed-mode fatigue crack growth. Materials science, 45: 190 (2009).

13. D. Rozumek, E. Macha, J-integral in the description of fatigue crack growth rate induced by different ratios of torsion to bending loading in $\mathrm{AlCu} 4 \mathrm{Mg} 1$. Materialwissenschaft und Werkstofftechnik, 40: $743-749$ (2009) 